\title{
ADAPTIVE NEUROFUZZY SYSTEM FOR ASTHAMA
}

\author{
Shashank Bhardwaj ${ }^{1}$, Niraj Singhal ${ }^{2}$, Neeraj Gupta ${ }^{3}$ \\ ${ }^{1}$ Shobhit University, Meerut, India \\ ${ }^{2}$ Shobhit University, Meerut, India \\ ${ }^{3}$ KIET, Ghaziabad, India
}

\begin{abstract}
The problem of health monitoring has been taken as it is one of the challenging problems in rural areas where people many times do not get proper treatment and are not financially sound to visit doctors in city. Asthma is a prevalent disease; many lives are lost due to lack of proper treatment which in turn can be saved if proper prognosis is done in time. In this study of neuro fuzzy network, a detailed study has been done on the various schemes and strategies that are part of neural networks.

The world today is not based only on discrete concepts but on fuzzy concepts, which made us research on the world of soft computing and finally a health monitoring system based on neural network is developed. Our objective is to study the various techniques and algorithms of neural network and to find the most efficient technique to implement this problem of health monitoring. The estimation of information about disease is based on the variables that affect its state.
\end{abstract}

\section{INTRODUCTION}

Fuzzy Logic has emerged as one of the active area of research activity particularly in control system application. Fuzzy logic is a powerful method of reasoning when mathematical models are not available and input data are imprecise. Its applications, mainly to control are being studied throughout the world by control engineers. Wherever logic in the spirit of human thinking can be introduced, fuzzy logic finds extreme application there. Sacrificing some amount of information we get a more robust summary of the information. What this really mean? Though we are conditioned to think in precise quantities, at a subconscious level, we think and take actions that are fuzzy in nature. And that is the way we perceive the nature and react to it.

Before going to into the details, let's look how fuzzy logic has become a household jargon. Though fuzzy logic originated in USA some 30 years back, the researchers there were skeptic about its applicability in real world applications and some even scoffed it off as nothing but probability. On the other hand, the Japanese was closely the pioneering work done by Mamdani and his associates in steam engine control and started applying fuzzy control even too consumer goods like cameras, air conditioners, vacuum cleaners etc. Thus fuzzy based products became highly competitive due to better performance, high reliability, robustness, low power consumption, cheapness etc. One of landmark success of fuzzy control was complete automation of subway train's drive control system in Japan. With fuzzy logic getting a wider acceptance in recent years, it is predicted that by the end of decade fuzzy logic will replace most of convention logic. Many projects which were nearly impossible earlier are now finding a new way out by fuzzifying them.
In the classical control paradigm, much stress is laid on the precision of input, the intermediate steps that process them, and modeling of the system in question. In spite of this we observed that many a time such sophisticated classical controllers developed often find it difficult to perform in real world control problems. Because the real world is so complex that regardless of the complexity of our model of the problem and the care taken to design such models, there exist so many parameters that not been properly accounted for and many more of which are totally ignorant of. Whereas a fuzzy logic solution is tolerant to the imprecision in the inputs and the model of system and still produce an output that is desired out of the system. This was put in a more effective way by Lofti A. Zadeh, the father of fuzzy set theory, when he said "Most application of fuzzy logic exploit its tolerance for imprecision. Due to being costly precision, minimization of precision is needed for performing a task."

The thinking process involved in fuzzy realm is not complex. It is simple, elegant and easily applicable. The simplicity arises because it eludes mathematics to a great extent and elegance lies in its expressiveness. Even a person who does not know anything about camera operation can design fuzzy based controller for it with the help of expert camera operator.

\section{FUZZY SET VERSUS CRISP SET}

The main objective behind fuzzy logic is to represent and reason with some particular form of knowledge expressed in a linguistic form. However, when using a language oriented approach for knowledge representation, one has to build a conceptual framework to tackle its inherent vagueness. The traditional or crisp sets are based on a two value logic: objects are either members or not members. Every individual object is assigned a membership value $\mu$ of either 
1 or 0 that discriminates between members and non members of the crisp set as shown in red line in figure 1.

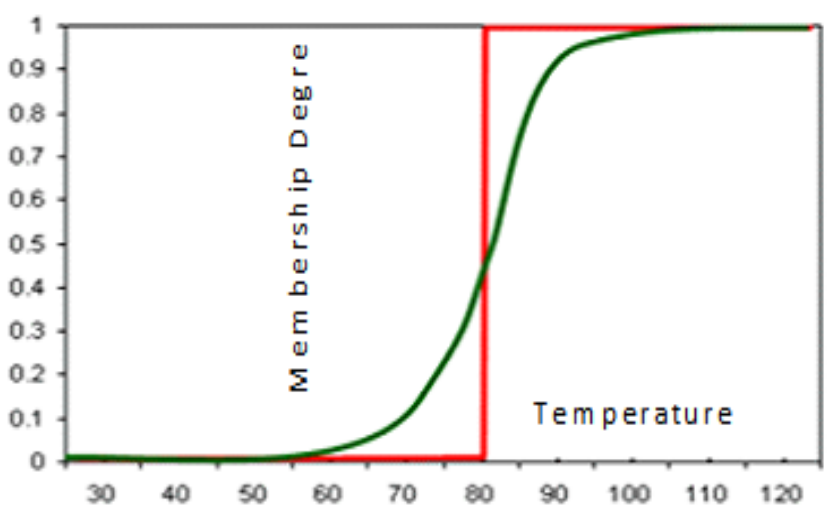

Fig 1 Crisp (red line) and fuzzy (green line) sets of "hot outside"

For example, the crisp set high in term of temperature may be defined such that:

$$
\mu_{\text {high }}=\left\{\begin{array}{l}
0 i f T<30^{\circ} \mathrm{C} \\
1 i f T \geq 30^{\circ} \mathrm{C}
\end{array}\right.
$$

Where $\mathrm{T}$ is the actual temperature. High is the linguistic variable that describes members of the set. The membership function $\mu_{h i g h}$ is the measure of "belongingness" to the category of High. If $\mathrm{T}=50^{\circ} \mathrm{C}, \mu_{\text {high }}\left(50^{\circ} \mathrm{c}\right)=1$, and the temperature is $100 \%$ high and definitely not low. Fuzzy-set theory on the other hand, is based on multivalued logic, and deals with the concept that are not sharply defined. In fuzzy logic membership in the linguistic variable High may be defined as:

$$
\mu_{\text {high }}=\left\{\begin{array}{l}
\text { Oif } 0 \leq T \leq 20 \\
\frac{T-20}{10} \text { if } 20 \leq T \leq 30 \\
\text { lif } 30 \leq T \leq 40
\end{array}\right.
$$

\subsection{Degree of Membership}

The degree of membership is the placement in the transition from 0 to 1 of conditions within a fuzzy set. The domain of a fuzzy set is the range of allowable values of the variable values of the variable as shown in green line in figure 1 . In this example the domain of a fuzzy set High is any value of $t$ from $0^{\circ} \mathrm{C}$ to $120^{\circ} \mathrm{C}$. The membership values $\mu$ high goes from zero (no membership) to unity (full membership) through intermediate values. A temperature of $25^{\circ} \mathrm{C}$ has a degree of membership of $(25-20) / 10=0.50$ in the fuzzy set High i.e. $\mu$-high $\left(25^{\circ} \mathrm{C}\right)=0.50$

The variable temperature may have many fuzzy sets associated with it (for example low, medium, high etc) and the domains of all the fuzzy sets constitutes what is known as the universe of discourse. The fuzzy sets low, medium and High associated with $\mathrm{T}$ may be defined as shown in fig 2.3. Here the universe of discourse is the range of values from 0 -c to $40^{\circ} \mathrm{C}$. A temperature of $22^{\circ} \mathrm{C}$ belong to the set low to a degree of $0\left(\mu \operatorname{low}\left(22^{\circ} \mathrm{C}\right)=0\right)$, to the set medium to a degree of $0.8\left(\mu\right.$ medium $\left.\left(22^{\circ} \mathrm{C}\right)=0.8\right)$ and to the set High to a degree of $0.2\left(\mu \operatorname{high}\left(22 \mathrm{C}^{\circ}\right)=0.2\right)$.

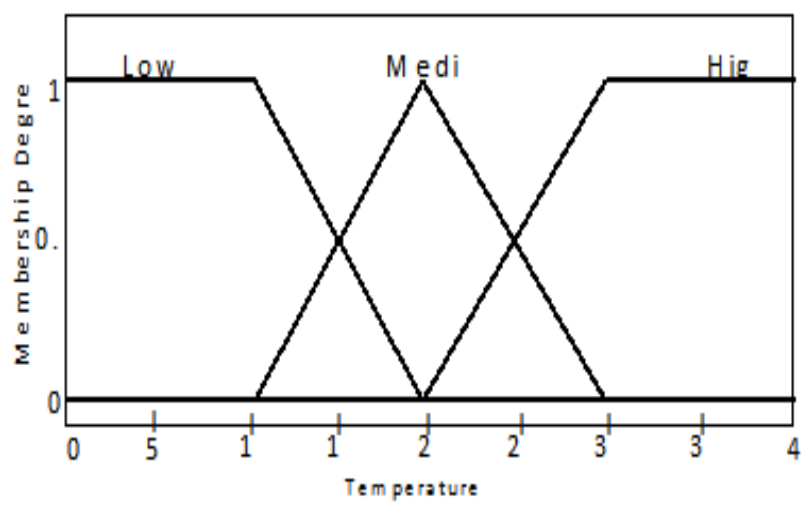

Fig 2 Membership Functions of Temperature

As illustrated in Fig 2, the features of the triangular membership function has been presented.

\section{ASTHMA}

Asthma is a very common disease that may become lethal if remained untreated. An asthma patient must always be alert with their body condition, especially their respiratory and the surroundings they are in. This paper proposes a development of a rule-based asthma system. This system provides advice for patients who would like to know the chances of getting an asthma attack are based on the patient's current asthma condition and surrounding environment. To do so, the patients will answer questionnaire to enable the system to understand their current health condition and the environment they are living in. It will then provide suggestions to avoid the attack, or to hasten asthma recovery, which includes environmental factors. Data regarding asthma was gathered through an interview with doctors and online medical resources. A rulebased algorithm was developed based on the data gathered. With this program, it allows patient's self management to lead a healthy, asthma-free lifestyle.

Although anyone may have an asthma attack, it most commonly occurs in:

- $\quad$ Children and adolescents ages 5 to 17 years

- $\quad$ Females

- People living in urban communities

- People having high intake of alcohol and smoke

\subsection{Input and Output Variables of Asthma}

Input variables are:

- $\quad$ Age: it has three membership functions as

- young age

- $\quad$ adult age 
- old age

- $\quad$ Gender: it has two membership functions as

- male

- female

- $\quad$ Economic status: it has two membership functions as

- Low

- high

- Tobacco and smoke consumption: it has three membership function

- Low

- Medium

- high

Output variable is:

- Asthma have three membership function

- low

- medium

- $\quad$ high

\subsection{If - Then Rules for Controller.}

The fuzziness of a fuzzy membership permits us to handle the problem of disease prognosis, we have defined various membership functions based upon the factors that are responsible for respective diseases. Combining the various research data about the diseases we have laid down linguistic fuzzy rules of the form:

- If (age is young_ones) and (gender_age is female) and (economic_status is low) and (tobacco and somke is low) then (output is low).

- If (age is young_ones) and (gender_age is female) and (economic_status is low) and (tobacco and somke is high) then (output is high).

- If (age is young_ones) and (gender_age is female) and (economic_status is high) and (tobacco and somke is high) then (output is high).

- If (age is young_ones) and (gender_age is male) and (economic_status is low) and (tobacco and somke is low) then (output is medium).

- If (age is young_ones) and (gender_age is male) and (economic_status is low) and (tobacco and somke is high) then (output is high).

- If (age is young_ones) and (gender_age is male) and (economic_status is high) and (tobacco and somke is high) then (output is high).

- If (age is adults) and (gender_age is female) and (economic_status is low) and (tobacco and somke is medium) then (output is medium).

- If (age is adults) and (gender_age is female) and (economic_status is high) and (tobacco and somke is high) then (output is high).

- If (age is adults) and (gender_age is male) and (economic_status is low) and (tobacco and somke is low) then (output is low).

\subsection{Simulation Results}

\subsubsection{FIS EDITOR}

The FIS(fuzzy logic inference) Editor is the high-level display for fuzzy logic inference system as shown in fig 4 .

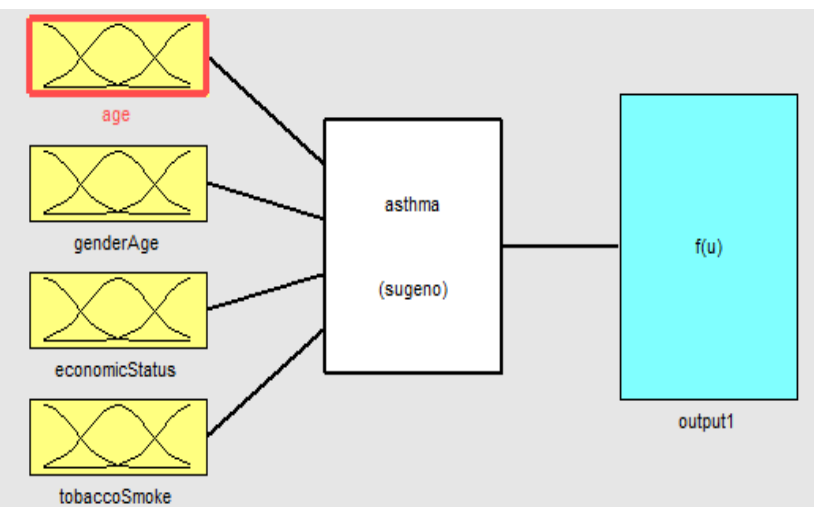

Fig 3 FIS EDITOR

\subsubsection{Membership Function of 'Age'}

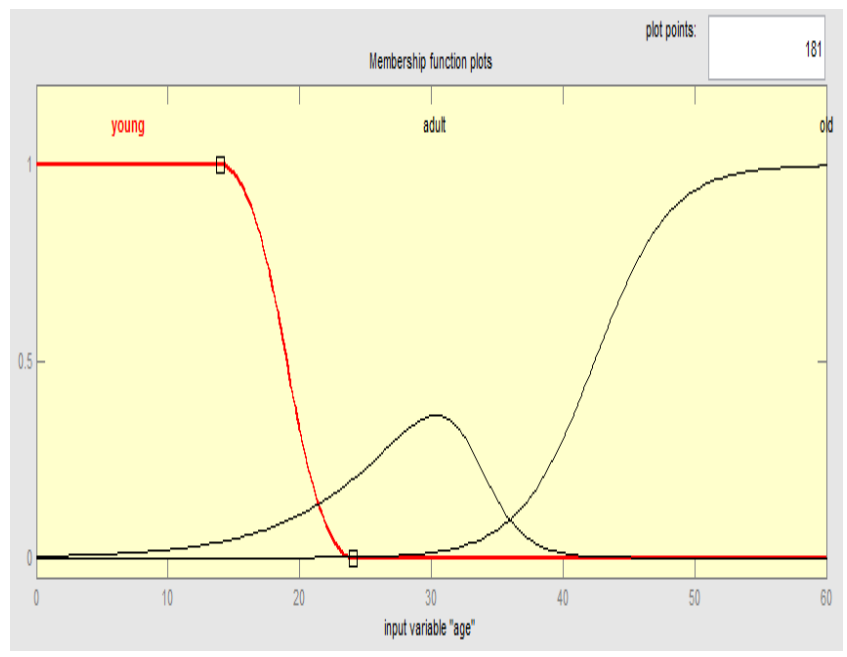

Fig 4 Membership Function of 'age'

\subsubsection{Membership Function of 'Gender'}

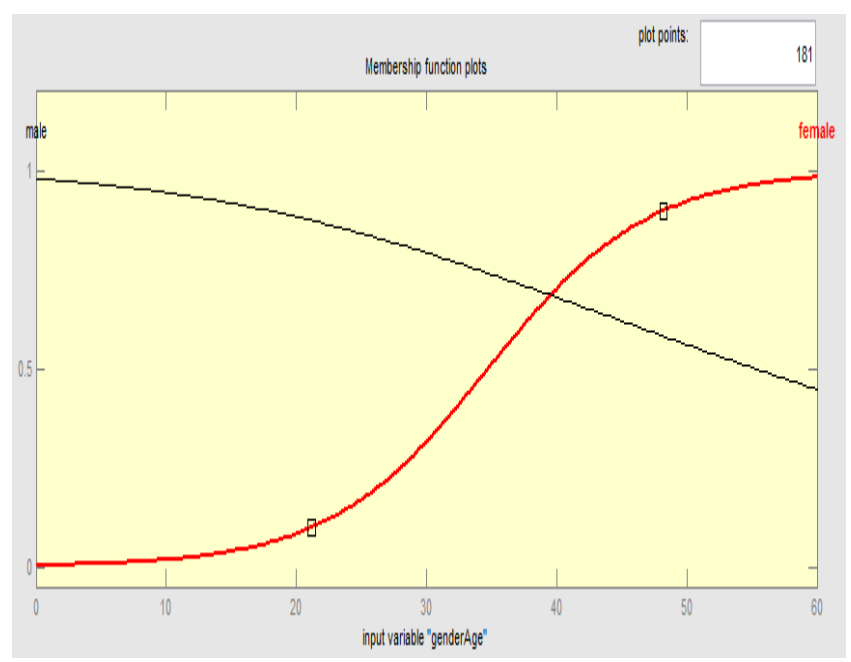

Fig 5 Membership Function of 'gender' 


\subsubsection{Membership Function of 'Economic Status'}

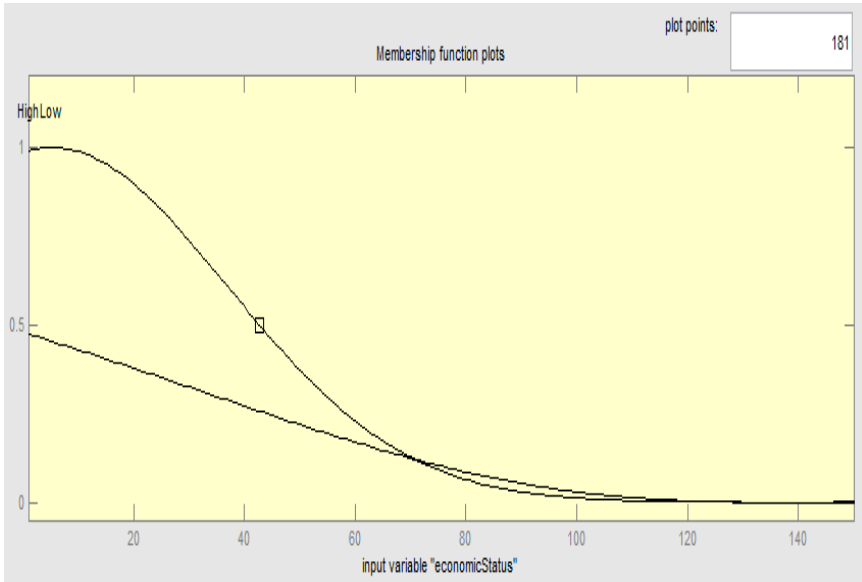

Fig 6 Membership Function of 'economic status'

\subsubsection{Membership Function of 'Tobacco and Smoke}

\section{Consumption'}

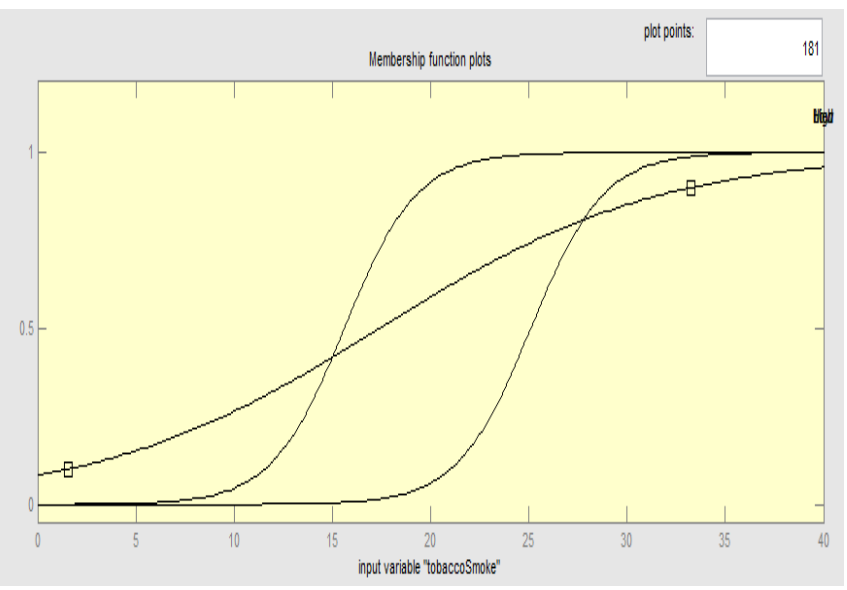

Fig 7 Membership Function of 'tobacco and smoke consumption'

\subsubsection{Rule Viewer of Asthma}
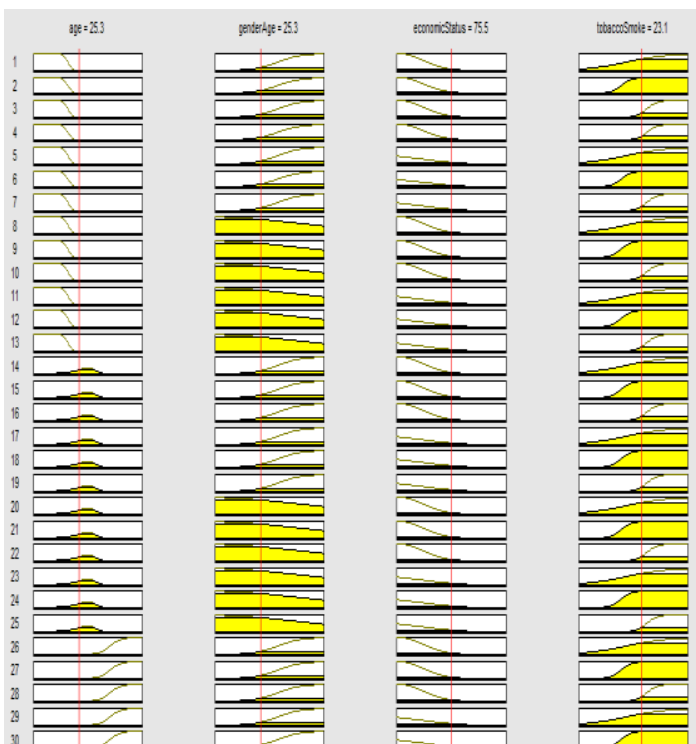

Fig 8 Rule viewer

\subsubsection{Surface Viewer before Training and Testing}

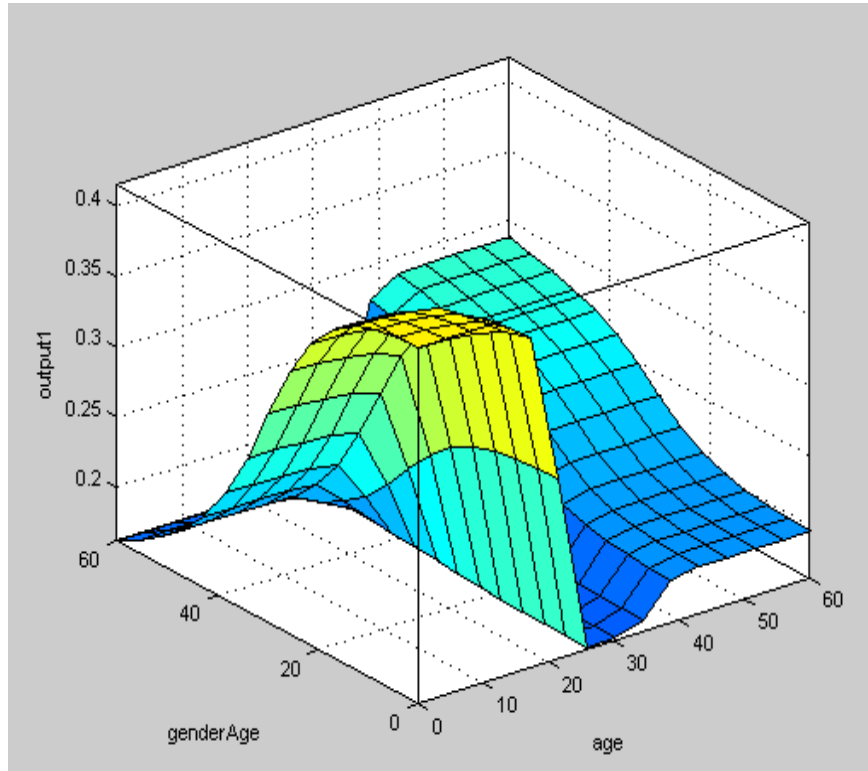

Fig 9 Relationship between 'age' and 'gender age'

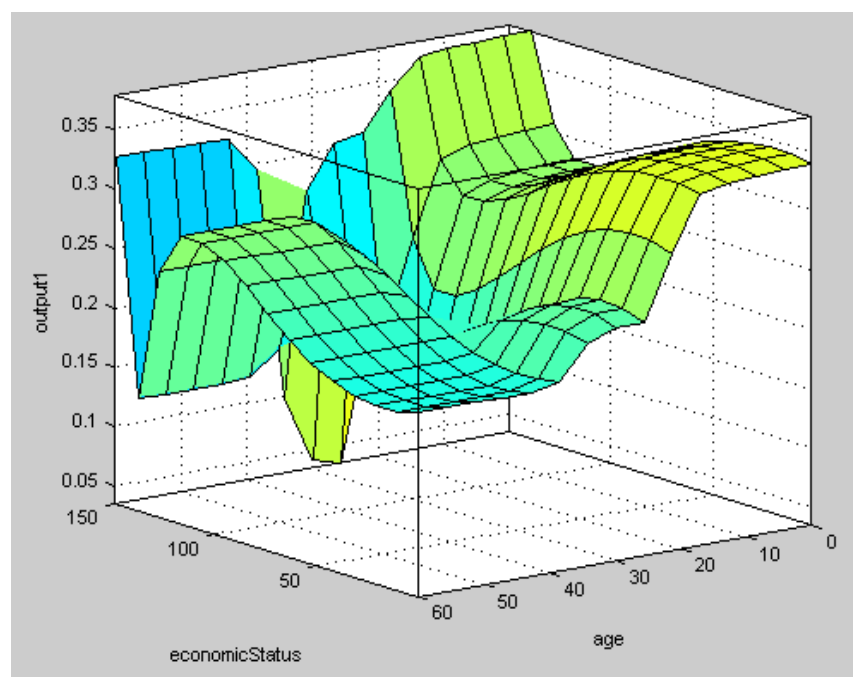

Fig. 10 Relationship between 'age' and 'economic status'

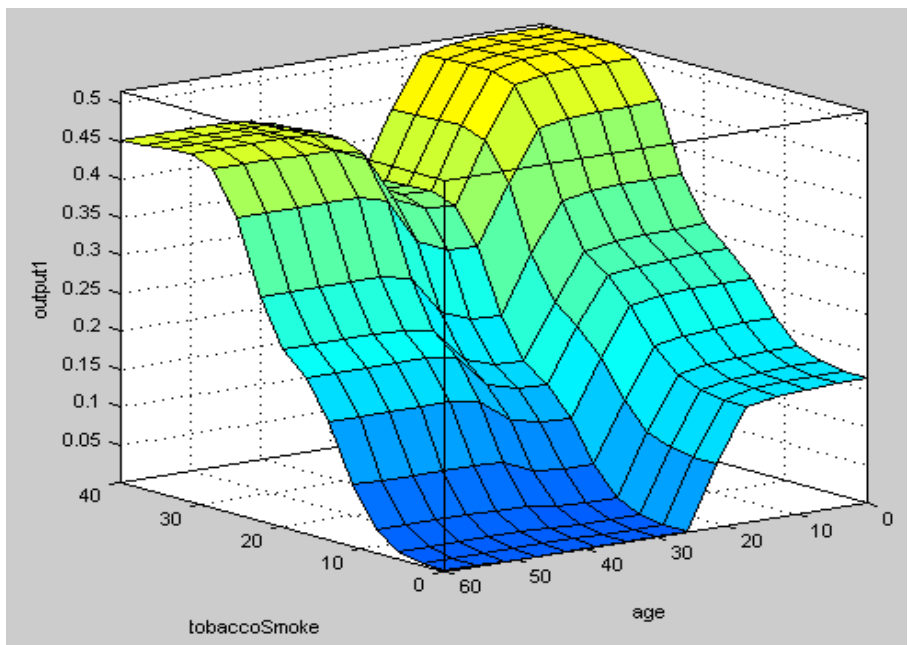

Fig 11 Relationship between 'age' and 'tobacco and smoke consumption' 


\subsubsection{Surface Viewer after Training and Testing}

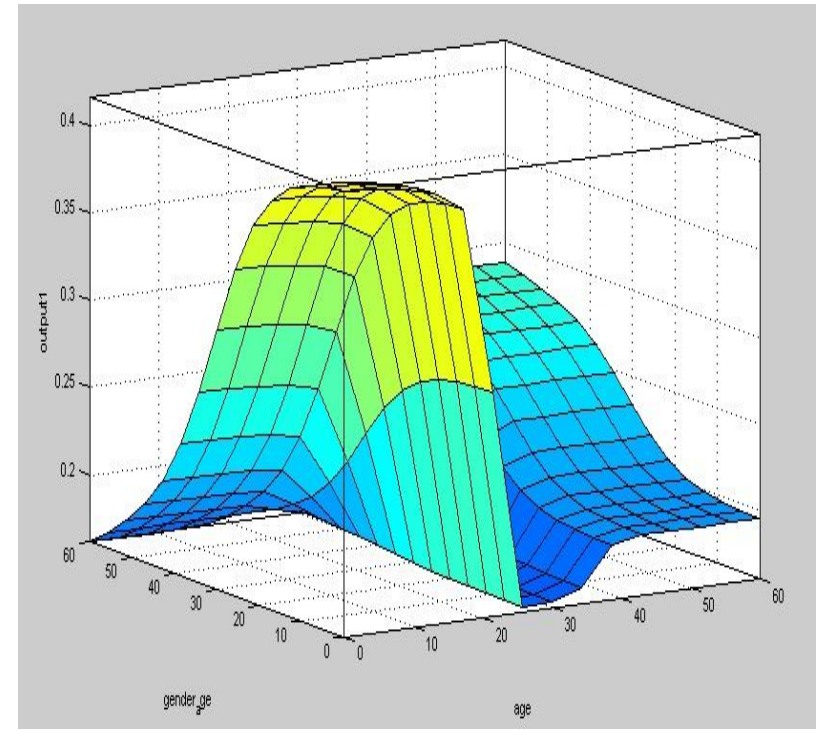

Fig 12 Relationship between 'age' and 'gender age'

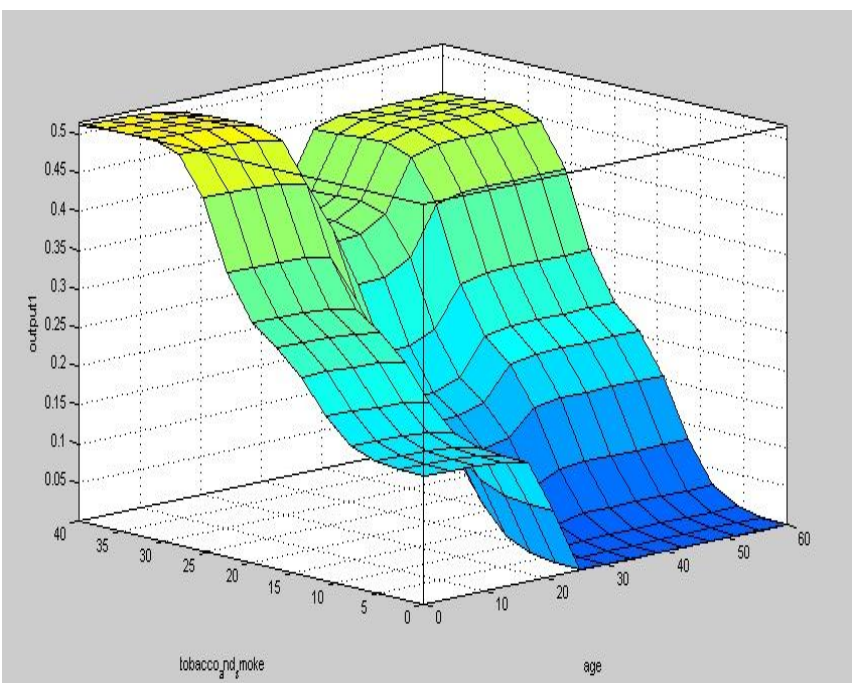

Fig 13 Relationship between 'age' and 'tobacco and smoke '

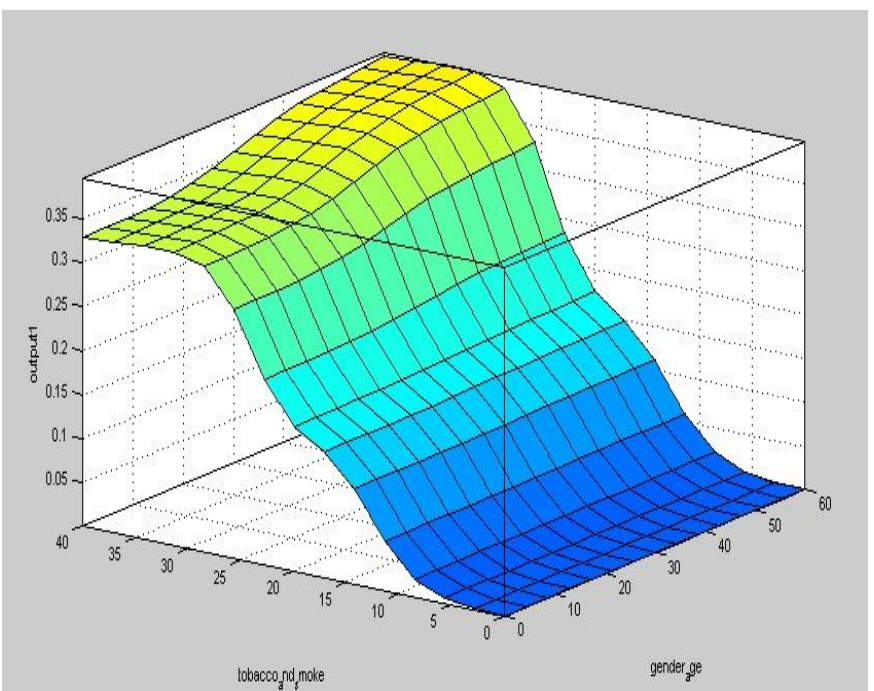

Fig 14 Relationship between 'gender age' and 'tobacco and smoke'

\subsubsection{Error during Training}

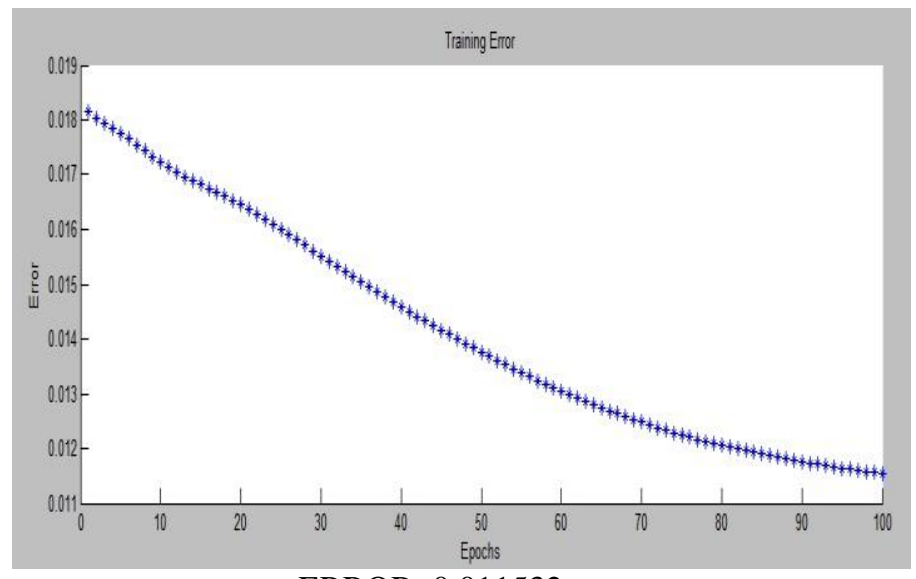

ERROR: 0.011532

Fig 15 Decrement in Training Error

\subsubsection{Neural Network Model of Asthma in}

\section{MATLAB Software}

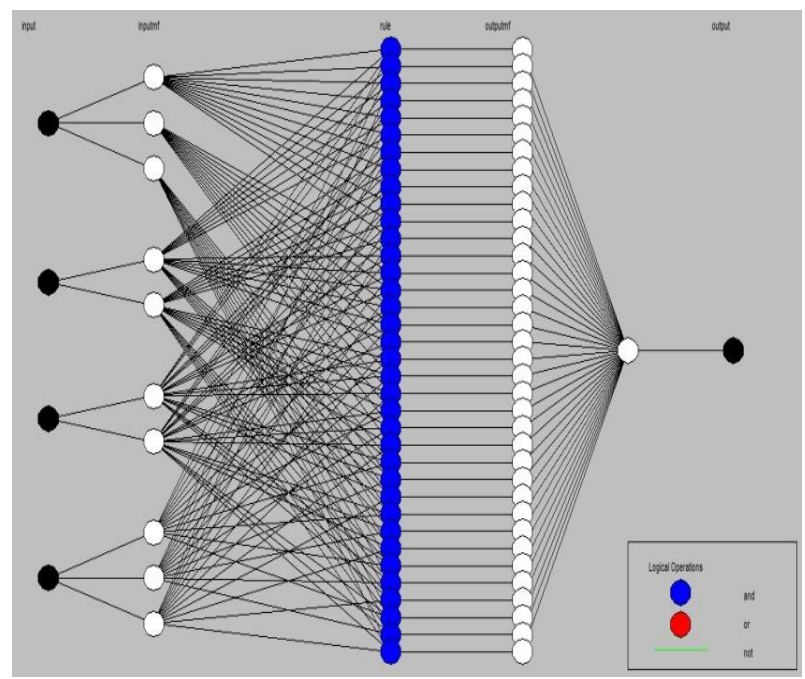

Fig 16 ANN Model of Asthma

\section{CONCLUSIONS}

In this project work the detailed analysis of Asthma was carried out considering the various input factors and its symptoms. The analysis was carried out using the Neuro Fuzzy Toolbox of the Matlab program.

This analysis in India will help the unprivileged and the rural section of the Indian Society overcome the burden of unnecessary tests and medicines associated with this disease. This work will prove to be helpful for all those people who cannot afford to consult the expert doctors owing to their high fees and unavailability in the rural areas. This project work has a promising future in the field of the Neural Network and Fuzzy Logic in the designing of a complete model comprising of the analysis of a number of common diseases in India. Once globalized, this study will help reduce the global burden of Asthma. 


\section{REFERENCES}

[1]. B. Kosko, Neural nets and Fuzzy Systems, Prentice Hall, 1992.

[2]. A. Q. Ansari and Neeraj Gupta, Design and Simulation of OTA-Based Activation and Membership Function for Neurofuzzy Systems, IJCC, VOL. 9, NO. 1, MARCH 2011

[3]. G. Castellano, C. Castiello, A.M. Fanelli1 and L. Jain, Evolutionary neuro-fuzzy systems and applications

[4]. A. Q. Ansari, Neeraj Gupta, Ekata, Automatic Diagnosis of Asthma Using Neurofuzzy System, 2012

Fourth International Conference on Computational Intelligence and Communication Networks

[5]. Neural-Fuzzy Applications in Computer Vision, Journal of Intelligent and Robotic Systems 29: 309-315, 2000

[6]. Neuro-Fuzzy and Soft Computing, http://mirlab.org/jang/book/

[7]. http://www.csee.wvu.edu/classes/cpe521 /presentations/ANFIS.pdf

[8]. Fuzzy Logic Toolbox: http://radio.feld.cvut.cz/ matlab/ toolbox/ fuzzy/fuzzyt 12.html

[9]. L. A. Zadeh, "Biological applications of the theory of fuzzy sets and systems," in Proc. Int. Symp. Biocybernetics, Central Nervous Syst., 1969, pp. 199-206.

[10]. R. N. Shiffman, "Towards Effective Implementation of a Pediatric Asthma Guideline: Integration of Decision Support and Clinical Workflow Support," Journal of the American Medical Informatics Association, pp. 797-801, November 1998. 